\title{
Application Limitations and Future Development Directions of Air Hammer Drilling Technology
}

\author{
HU Gui *, HUANG Xueqin, LIU Xinyun, MENG Qingkun \\ PetroChina Research Institute of Petroleum Exploration \& Development, Beijing, China
}

\begin{abstract}
Air hammers, also called Down-The-Hole hammers, are previously applied in mining industry, civil engineering, hydrology well drilling and have long been considered an effective approach to improve the rate of penetration (ROP) especially in hard formations. The development of air hammers suitable for oil \& gas deep well drilling industry in 2000s substantially increase the drilling ROP in hard to mediate hard formations and effectively prevent well deviation in high dip formations. However, its expanding application in oil \& gas field also shows that some problems still exist in following operation: drilling casing accessories, drilling in the wet or water formations, drilling in sloughing wellbores. This paper discusses these application limitations, presents the possible resolutions and future development directions which may help to extend the hammer drilling application range and level.
\end{abstract}

\section{Introduction}

Compared to conventional rotary drilling with tri-cone or Polycrystalline Diamond Compact (PDC) bits, percussion tools can increase Rate of Penetration (ROP) by 2-15 times in hard rock [1,2]. In civil engineering, hydrologic geology and mining industries, air hammer, also called Down-The-Hole (DTH) hammer, a kind of percussion tool used in air drilling, has been used to drilling shallow wells in hard rock formations from 1950s [3,4].

In 1980s, the air drilling technology developed rapidly in oil \& gas drilling industry and could increase the ROP more than 5 times using tri-cone bits with high Weight on Bit (WOB) (more than 3 tons, average 10 tons) owing to the reduced chip hold down effect on rock $[5,6,7]$ with air drilling medium. Nevertheless, high WOB benefit the ROP but easily induce well deviation. A new kind of percussion drilling tool with low WOB is needed and that is air hammers. Therefore, a new kind of Deep Well Air Hammers (DWAH) suitable for deep well drilling characterized high temperature, high Air Consumption (AC) and high longevity are developed from 2003[1].

DWAH is suitable for drilling in depth of $0 \sim 5,000$ meters. Its Air Consumptions (AC) is more than 80 cubic meters per minute which contributes a lot to the cuttings transportation. The down-hole working longevity is more than 120 hours with a longest drilling section of 1950 meters. Applications in oil \& gas field showed this tool had great superiority in improving Rate Of Penetration (ROP) (10 times more than traditional mud drilling, 2 5times more than air drilling with tri-cone bits), preventing hole deviation, speeding the petroleum exploration and development. Up till now, the air hammer has drilled more than 250,000 meters in Chinese southwest oil \& gas field.

Although, air hammer increases ROP a lot and controls the well deviation effectively, it is not always all round. In ultra-hard formations, borehole sloughing formations, wet or water formations, directional drilling sections, air hammer drilling technique is still not suitable or has no obvious ROP improvement. This paper introduces air hammer drilling technique, discusses the limitations and possible future development directions of air hammers in formations listed above.

\section{Specifications of DWAH}

The DWAH work mechanism is the same to the DTH hammer in shallow wells, but has the following Characteristics: (1)High AC: with the needing ability to transport cuttings from more than 3,000 m depth and to blowout the sloughing debris in case of trouble zones such as coal formations, the air consumption is increased to more than $80 \mathrm{~m}^{3} / \mathrm{min}$ which is 2 times volume of shallow well hammers. In some ultimate circumstance, the feeding air volume can get to $220 \mathrm{~m}^{3} / \mathrm{min}$. (2)High bit gauge protection ability: hammer bits are always required to drilling a long section with high ROP within one tripping. So the bit needs to increase the gauge protection ability. A bit with low gauge protection ability would drill a shrink hole which would cause the following tripin difficulties. (3)Long longevity: in shallow wells, the total depth is always no more than $1,000 \mathrm{~m}$ and the working time is always no more than $50 \mathrm{~h}$. However, in petroleum industry, a section more than 2,000 m always needs long drilling time. Thus, the longevity of air hammer needs to improve to more than $100 \mathrm{~h}$.

The drilling stand pipe pressure (SPP), WOB, Rotary Speed (RS), AC parameters and specifications of DWAH are showed in table 1 .

Table 1. Specifications of DWAH

\begin{tabular}{|c|c|c|c|c|c|c|}
\hline $\begin{array}{l}\text { Hammer } \\
\text { OD } \\
\mathrm{mm}\end{array}$ & $\begin{array}{l}\text { Bit } \\
\text { OD } \\
\text { inch }\end{array}$ & $\begin{array}{l}\text { We- } \\
\text { ight } \\
\mathrm{kg}\end{array}$ & $\begin{array}{c}\text { WOB } \\
k N\end{array}$ & $\begin{array}{c}\mathrm{RS} \\
\mathrm{r} / \mathrm{mi} \\
\mathrm{n}\end{array}$ & $\begin{array}{l}\text { SPP } \\
\mathrm{MPa}\end{array}$ & $\begin{array}{c}\mathrm{AC} \\
\mathrm{m}^{3} / \mathrm{mi} \\
\mathrm{n}\end{array}$ \\
\hline 275 & 17.5 & 618 & & \multirow{6}{*}{$\begin{array}{c}30- \\
50\end{array}$} & & \\
\hline 275 & $\begin{array}{c}12.2 \\
5 \\
1 / 4 \\
\end{array}$ & 618 & $30-50$ & & $\begin{array}{c}1.8- \\
2.5\end{array}$ & $\begin{array}{l}90- \\
150\end{array}$ \\
\hline 180 & 9.5 & 277 & \multirow{2}{*}{$20-30$} & & \multirow{2}{*}{$2-3$} & 75- \\
\hline 180 & 8.5 & 277 & & & & 120 \\
\hline 135 & 6 & 106 & $20-30$ & & $2-3$ & $48-80$ \\
\hline 90 & 4.5 & 86 & $15-25$ & & $2-3$ & $30-80$ \\
\hline
\end{tabular}




\section{Limitations and possible future development directions of air hammer drilling technology}

\subsection{Operations of long-term reaming}

Long-term reaming Operations using air hammers are disallowed. Reaming is the solution of well under-gauge. The bit of air hammers has no cutting inserts, but cylindrical and composite ball teeth which are impact teeth. If the air hammers start to work during the reaming operation, the bit and rocks would not contact completely. The impulse energy cannot be transmitted directly and would cause the vibration of bit. This would aggravate the broken or the wear of teeth (see Fig. 1). Thus, longterm reaming would wear the bit teeth rapidly and affect the transmission or rock fragmentation efficiency of the impacts stress wave after the air hammers down to the well bottom.

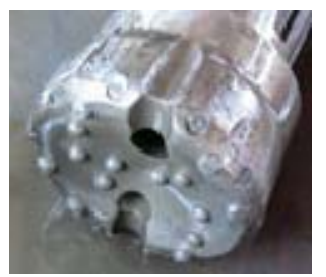

Fig. 1 Worn Bits of Air Hammer

\subsection{Drilling in ultra-hard formations}

Most air hammers used in oil \& gas field drill is currently not suitable for ultra-hard formations, and is also not suitable for drilling in casing accessories. When forced into such operations, the ball inserts may be damaged drastically and the matrix may be worn speedily. Both the results affect the bit's longevity and penetration rate. In one application case, the air hammers was employed to drill the casing accessories to save one drilling string trip. After operation, the ROP decreased rapidly, only $4 \sim 5 \mathrm{~m} / \mathrm{h}$ compared to $10-15 \mathrm{~m} / \mathrm{h}$ in normal conditions.

According to the structure of the bit face, there are three types of bit face [8]: flat, concave and convex cone face. Typically, the drillable strata hardness relationship among them is following: flat face bits < concave cone face bits < convex cone face bits. Bits with flat face are suitable for drilling in soft to medium hard formations; and bits with concave cone face are able to drill most medium hard to hard strata; while bits with convex cone face are mainly used in ultra-hard formations. Presently, most air hammer bits need to drill a long section, and most of the formations drilled are medium hard to hard. Therefore the bits always adopt structure of concave cone face. In oil \& gas field, we should use roller bits to drill the casing accessories; when drilling in ultra-hard layers we should understand lithology completely and choose structure of convex cone face bits if necessary. This kind of bit can generate large impacting power in a smaller area and obtain high drilling rate. However, the ability of drilling a well vertically may not be so well compared to flat and concave cone face bit.

\subsection{Drilling in sloughing formations}

Borehole collapse is a challenge for drilling operators. If the tri-cone bits were chosen for the air drilling, borehole collapse can be relieved by back-reaming, high torque rotation of drilling string and lifting the drilling string up and down with high gas injection rate. Since the air hammers have no back-reaming ability, once encounters borehole sloughing, the only measure is increasing gas injection rate to blowout the sloughing debris. And this is unsafe if the highest gas injection rate (always no more than $250 \mathrm{~m}^{3} / \mathrm{min}$ owing to the air compressors' capacity) is not enough to blowout the all the sloughing debris. Later, a serious drill pipe frozen may occur. Usually, under such circumstances, operators give up the air hammers drilling and select the air drilling with tri-bits or traditional mud drilling.

Air drilling is a kind of underbalanced drilling. It is inevitable to induce borehole collapse due to the high under-pressure situation. No capacity of processing the hole-caving problems in air drilling limits the wide application of air hammers. Therefore, a new type of air hammers with ability of handling the trouble of well collapse should be developed.

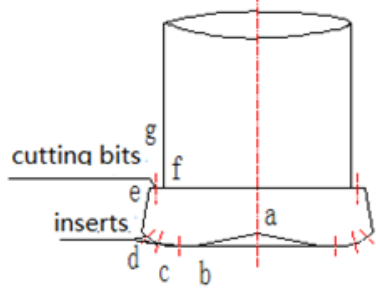

Fig.2 Scheme of air hammer bit with back-reaming ability

Patent [9] proposes a bit structure of air hammers capable of back-reaming (see Fig. 2). Certain amounts of cutting teeth are inserted on the back of air hammers' bit. Normally, the cutting teeth do not work. When the hammer bit is in shrinkage wellbores, trip out the drilling strings to the shrink point and rotate the drilling string and start to back-ream. This kind of air hammers' bit structure provides a solution to the operation in sloughing formations, however, much work still needs to be done.

\subsection{Drilling in wet or water formations}

When Air drilling technology are applied in wet or water formations, the powdery cuttings are extremely easy to absorb the water and paste together on the drilling tools, especially on the bits; form a pie of "mud collar" and seal off the annulus between drilling strings and wellbore; at last cause the SPP to increase and the AC to reduce. In serious situations, the moisten cuttings would block the flowing channel of gas, causing the failure of air drilling operation. Then the operators need to change into misted drilling, foamed drilling or mud drilling according the amount of water produced. If the bit were tri-cone bit, the change of medium would be convenient since there was no necessity of bit-change. If the drilling tools are air hammers, the change of medium would cause the failure of air hammers and necessity of bit-change. Literature [10] illustrates that the influences of well bottom pressure 
(WBP) on the internal normal work of air hammers. When the equivalent density of drilling medium in annulus increases, the energy output of air hammers decreases. In misted/foamed drilling, the equivalent density of drilling medium is higher than that of gas and so the WBP is higher. In water formations, the gas need to blowout the water. The added water in annulus also increases the WBP. Both two listed above would lead to the lower energy output of air hammers, and lower drilling ROP. Except for the lower energy output of air hammers in wet formations, reservoir fluids backflow into the air chamber of air hammers is another problem. When stopping gas circulation during the pipe connection time, the water combined with the cruds from formations gathers into the hole and backflow into the air chambers through the bit nozzles. The cruds easily contaminate the air chambers and cause the failure of air hammers.

Therefore, operators are careful to select the air hammers drilling technology if the target section imbeds with wet or water layer. Such application situations limit the wide use of air hammers.

Three measures may solve this problem: misted hammers, foamed hammers, and reverse cycle air hammers drilling technology. The test of air hammer drilling in misted \& foam drilling medium shows that the air hammers only drilled a short section (no more than $100 \mathrm{~m}$ ) with a low ROP (smaller than $5 \mathrm{~m} / \mathrm{h}$ ) before its failure[10]. Through the study on the working mechanism of air hammers, we found that the key point of misted or foamed hammers device is how to guarantee the air hammer impact power output when the WBP increases. The hammers must self-adapt the shift of WBP and keep a high impulse energy output, thus, a new kind of foam hammer should be developed [11].

Reverse cycle air hammers drilling technology [3,4], is another method to solve the drilling problem in water formations. It refers to an engineering that carry the drilling cuttings from the drill centre to ground. This drilling method has been employed in shallow well drilling such as civil engineering, hydrologic geology and mining industries. Whether it can server in petroleum drilling is still in the theoretical and experimental study period [12]. The advantages of reverse cycle air hammer drilling technology are following: (1) good cuttings carrying ability and high ROP; (2)good adaptability to the wet or water formation. With high gas flow velocity, all the water can carry from the inner hole of drilling string effective and have no impact on the wellbore. (3)lower trouble risk. There is no washing on the wellbore with gas or water from the wet formation, thus the wellbore stability is good with low troubles risk. However, the reverse circulation drilling technology is totally different from normal circulation cycle, many traditional drilling tools need to redesign, such as wellhead equipment, BOPs, RAMs, swivels, double wall drill pipes. The most challengeable tools may be the double wall drill pipes suitable for deep wells. Some work has been done on these pipes but with no effective progress.

\subsection{Drilling in directional wells}

Air hammers are not only able to increase the ROP and control the well deviation in vertical wells, also are capable of applying in directional wells. Presently, more and more wells are directional wells and need directional well ROP enhancing means. Air hammers are previously thought to be suitable for drilling in vertical wells. Literature [13] shows that: because of the low WOB, the influences of rock bit's side force on drilling direction are small. The drilling direction mainly depends on the direction of normal line of bit bottom face. If the air hammers were well assembled with directional tools, by adjusting the normal line of bit bottom surface, the air hammers are able to be employed in directional drilling.

There are two means of directional drilling with air hammers. First efforts to drill directional wells with an air hammer included a directional or self-rotates air hammer [14] in which the piston was designed to rotate and impact on the hammer bit as it oscillates inside the hammer. This technology type has been used primarily in well inclined of 30 degrees or in " $S$ " shaped directional wells. Another approach is running a packed-hole BHA to maintain inclination after the curve was built with a motor BHA. A number of high-angle directional wells were drilled successfully with this method in the Granite Wash formation in the Texas Panhandle in 2005-2006.

However, directional drilling with air hammers has not yet been applied in China. It it was restricted by the development of efficient gas PDM and reliability of selfrotary air hammers. In fact, the world's directional drilling with air hammers is also not so widely applied. Much work is still needed to be done.

1. Measurement While Drilling (MWD) in gas drilling medium. To control the well direction, we need to know the trajectory parameters when drilling. Thus survey is important. However, traditional MWD only works in mud medium. In gas medium, presently, the only mean for survey is single shot survey which is too simple, and inefficient. Another possible approach is Electromagnetic MWD (EM-MWD). Lots of efforts has been put on the research of EM-MWD and currently it still need to be improved $[15,16]$.

2. The low-speed and high longevity gas motors. Motors can drive the bit rotate without the drive of drilling string. Most of the gas PDMs rotary speed are high [17] and cannot meet the air hammers requirement of 30-50 r/min rotary speed. Thus the low-speed and high longevity gas motors is still need to be developed.

3. Air hammers with ability of directional drilling. Under the condition of deviated and horizontal wells, the kinematic characteristics of the piston of Air hammer change a lot [10]. The lying piston in the cylinder (the centre and the piston gravity has no or little changes when the piston moves) reduces the performance of piston energy storage and intensify the friction between piston and cylinder. These two influence the working performance of air hammer.

4. Self-rotary air hammers. Self-rotary air hammers $[18,19]$ are sensitive to the drilling parameter of WOB. The WOB easily influences the energy output and even the reliability of self-rotary air hammer. Little literature 
shows their relationships, and much work still needs to be done.

\section{Conclusions}

1. Air hammers drilling technology cannot applied in long-term reaming operation. The long-term reaming operation would wear the bit rapidly.

2. The air hammer with bit of concave cone face do not adapt to ultra-hard strata and the casing accessories. When meets such formations, we should select the bit with convex cone bit face.

3. Bit with ability of back-reaming should be further developed to meet the drilling requirements in sloughing formations.

4. Misted and foamed hammers adaptive to the increases of bottom hole pressure need to be further studied and developed.

5. Air hammers drilling in directional wells has been applied in some blocks but not widely used. EM-MWD, gas MWDs, gas motors, air hammer with ability of directional drilling and self-rotary air hammer still need to be improved and advanced.

\section{References}

[1] MENG Qingkun, WANG Xiangdong, YU Xingsheng. Application of KQC Series of Air Hammers for Gas Drilling in Oil Field [J]. Oil Field Equipment. 2007.36(11) 54-57

[2] M.C. Whiteley , W.P. England, Air Drilling Operations Improved by Percussion Bit/Hammer Tool Tandem, Paper SPE-13429-MS, presented at 1985 SPE/IADC Drilling Conference, 5-8 March, New Orleans, Louisiana

[3] Geng Ruilun. Multi-process Air drilling technology [M]. Beijing: Geological Publishing House, 1995:1718.

[4] DU Xiangling. Down-to-hole Hammer Drilling technology [M].Beijing: Geological Publishing House,1988:1-4.

[5] YU Rui, XU Qicong, AI Jingtao, et al. Testing and Research on Gas Drilling Technology in Well Longgang 1 [J].Drilling \& Production Technology,2009,32(6):16-19.

[6] CHEN Jifeng,YAN Xiuliang,GAO Hangxian. Gas Drilling Technique in Northeast Sichuan Basin [J].Petroleum Drilling Techniques, 2009, 37(4):3942

[7] ZHANG Keqin,HOU Shugang. New progress of gas drilling integration and matching technologies in the Puguang Gas Field [J].Natural Gas Industry, 2010, 30(5):77-80.

[8] GENG Ruilun. Foreign DTH Hammer Bit Design and Effect $[\mathrm{J}]$. Exploration Engineering Translation Series, 1988 (3): 1-6.

[9] MENG Qingkun, WANG Xiangdong, HU Gui. Air Hammer Bit With capability to Back-ream, Chinese Patent, ZL201220257473.6 [P].2012-12-5
[10]HU Gui, MENG Qingkun, WANG Xiangdong, et al. Research on Operating Performance of Air Hammers in Foam Drilling [J]. Oil Field Equipment. 2009.38:(12):5-9

[11]Hu Gui,Meng Qingkun,Wang Xiangdong,Tao Ye. Research, development and application of a novel foam hammer capable of preventing downhole fluid backflow [J]. NATUR.GAS IND,2014, 34,I(7):71-74

[12]HAN Liexiang, SUN Haifang. Study and Development to Reverse Circulation Air-drilling Technology [J]. Drilling \& Production Technology,2008,31(5):1-5

[13]HU Gui, MENG Qingkun, WANG Xiangdong, et al. Application of Air hammer Drilling Technology in Directional Wells [J]. China Petroleum Machinery.2012.40(4):10-21,25

[14]Bui H., Meyers J. and Swadi S. Steerable Percussion Air Drilling System [C]. FETC Publications,1997 Conference Proceedings

[15]WANG Lei, LI Lin, SHENG Limin, et al.. Electromagnetic wave DREMWD system and its field test [J].Oil Drilling \& Production Technology,2013,35(2):20-23

[16]SU Yi, QI Xin, LIU Yang, et al.. Electromagnetic measurement while drilling technology based on the carrier communication principle [J].PETROLEUM EXPLORATION \& DEVELOPMENT, 2013,40(2):226-231.

[17]SHANG Li, XU Qicong, DENG Hu, et al..Output properties of PDM for gas drilling [J]. Fault-Block Oil \& Gas Field,2009,16(6):88-90

[18]HE Chao, HAN Liexiang, CHEN Xiaobin, et al.. Development of Air hammer with Self-propelled Round Bit [J]. Drilling \& Production Technology, 2011,34(1):63-64

[19]HE Chao, LI Jiang, HAN Liexiang, et al.. Failure Analysis of Key Components of Air Hammer with Self-propelled Round Bit [J]. Drilling \& Production Technology,2012,35(1): 66-68 